\title{
Vacuna Johnson \& Johnson contra COVID-19: distribución mundial de la vacuna, mecanismo de acción, indicaciones, contraindicaciones y efectos secundarios. Una revisión bibliográfica.
}

\author{
Johnson \& Johnson vaccine against COVID-19: worldwide \\ vaccine distribution, mechanism of action, indications, \\ contraindications and side effects. A literature review.
}

\begin{abstract}
Wilmer Gabriel Pineda-Palacios,* Jorge Andrés Rivas-Astudillo,* Geraldine Alexandra Saraguro-León,* Juan Andrés Solano-Espinoza,* Neyder Katherine Valdivieso-Tocto,* Diana Patricia Álvarez-Álvarez ${ }^{\ddagger}$
\end{abstract}

\section{RESUMEN}

El SARS-CoV-2, causante de que estemos viviendo una pandemia mundial, tuvo sus orígenes en China, desde donde ha traspasado fronteras rápidamente, llegando a todos los rincones del mundo. Muchos han sido los equipos de investigación que se enfrentan el reto de conseguir una vacuna que logre combatir este mortal virus. Es por este motivo que en esta investigación se pretendió analizar la bibliografía referida a la vacuna Johnson \& Johnson (J\&J) contra COVID-19: distribución mundial de la vacuna, mecanismo de acción, indicaciones, contraindicaciones y efectos secundarios. Varios estudios demuestran que su eficacia varía de acuerdo con la edad y género de cada individuo; sin embargo, esta vacuna alcanzó un grado de certeza moderada. Los efectos adversos en su mayoría son leves y se resolvieron al cabo de dos días, siendo excepción algunos casos, ya que se registró un efecto adverso poco común denominado trombocitopenia prevalente en mujeres de 18 a 40 años, por este motivo, la FDA (Administración de Alimentos y Medicamentos de EE.UU.) recomienda la precaución en el uso de la vacuna con respecto a este efecto adverso que en algunos casos podría ser mortal.

Palabras clave: SARS-CoV-2, coronavirus, vacunas, Johnson \& Johnson.

\section{ABSTRACT}

The SARS-CoV-2, which caused us to be experiencing a global pandemic, had its origins in China, from where it has crossed borders rapidly, reaching all corners of the world. Many research teams have faced the challenge of getting a vaccine to fight this deadly virus. For this reason, this research aimed to analyze the literature on the Johnson \& Johnson COVID-19 vaccine: global distribution of the vaccine, mechanism of action, indications, contraindications and side effects. Several studies show that its effectiveness varies according to the age and gender of each individual, but this vaccine reached a moderate degree of certainty. The adverse effects are mostly mild and resolved within two days, with some exceptions being a rare adverse effect called prevalent thrombocytopenia in women aged 18 to 40 years. For this reason, the FDA recommends caution in the use of the vaccine with respect to this potentially fatal adverse effect in some cases.

Keywords: SARS-CoV-2, coronavirus, vaccines, Johnson \& Johnson.

* Estudiante.

‡ Odontólogo. Docente Titular, Especialista en Endodoncia de la Universidad del Salvador.

Carrera de Odontología, Universidad Católica de Cuenca. Ecuador.

Recibido: 19 de agosto de 2021. Aceptado: 12 de septiembre de 2021.

Citar como: Pineda-Palacios WG, Rivas-Astudillo JA, Saraguro-León GA, Solano-Espinoza JA, Valdivieso-Tocto NK, Álvarez-Álvarez DP. Vacuna Johnson \& Johnson contra COVID-19: distribución mundial de la vacuna, mecanismo de acción, indicaciones, contraindicaciones y efectos secundarios. Una revisión bibliográfica. Rev ADM. 2021; 78 (5): 275-279. https://dx.doi.org/10.35366/102035 


\section{INTRODUCCIÓN}

A finales de diciembre de 2019 se notificaron los primeros casos de SARS-CoV-2, un nuevo tipo de coronavirus en la ciudad de Wuhan (China). El 28 de febrero la OMS la declaró como una emergencia global «muy alta». Desde entonces, el aumento de nuevos infectados por este virus que provoca la enfermedad denominada COVID-19 ha sido continuo y su transmisión de persona a persona se ha acelerado. En marzo de 2020, a raíz de los numerosos casos de brotes en el ámbito mundial, se tomó la medida de cerrar fronteras de manera obligatoria en la mayoría de países. ${ }^{1}$

Los coronavirus son pertenecientes a la gran familia Coronaviridae que tiene la capacidad de infectar a distintas especies, incluyendo a los humanos. Son virus formados de ácido ribonucleico (RNA) que no se encuentra segmentado, presentan envoltura y se componen de cuatro tipos de proteínas estructurales: la glucoproteína de membrana, la proteína de la envoltura, la proteína de la nucleocápside y la proteína S (spike) (glucoproteína espícula externa), ${ }^{2}$ siendo esta última la más importante de todas ya que se compone de dos subunidades, la primera de ellas se encarga de reconocer los receptores de la célula y la segunda permite su unión y con esto se origina el proceso infeccioso que empieza afectando las células de origen epitelial del sistema respiratorio, continuando con las células que intervienen en la formación de los alvéolos del pulmón. La sintomatología se caracteriza principalmente por la pérdida del gusto y del olfato, otros síntomas son el dolor de cabeza, tos seca, fiebre y en algunos casos diarrea. ${ }^{3,4}$

La comunidad científica se ha volcado en un mismo objetivo: encontrar una vacuna capaz de combatir la COVID-19. Varias han sido las farmacéuticas que se encuentran en la búsqueda de una vacuna eficaz y eficiente, que permita erradicar esta pandemia. Sin embargo, el reto no ha sido nada fácil, ya que es un virus completamente nuevo y desconocido para todos. Los fabricantes de las vacunas pueden basar su trabajo en cualquiera de estas plataformas: ${ }^{3-6}$

1. Inmunidad innata entrenada: las células constituyentes del sistema inmune innato sufren una nueva organización genética causada por un estímulo antigénico inespecífico. Ésta le permite al hospedador combatir eficazmente un segundo ataque del mismo patógeno o de otro. ${ }^{4}$

2. Basadas en ácidos nucleicos: se utiliza ácido desoxirribonucleico (DNA) o RNA modificados a nivel genético, los cuales tienen la capacidad de generar la proteína pico presente en la superficie del virus y ésta a su vez una respuesta inmunitaria que pueda combatir al SARS-CoV-2. Después esta proteína se ubica en la superficie de las células propias del cuerpo humano, el sistema inmune, al reconocer su ingreso, empieza a producir anticuerpos. Pfizer-BioNTech y Moderna crearon sus vacunas aplicando este técnica. ${ }^{2,3,5,6}$

3. Virus vivo: su empleo no está recomendado. ${ }^{5}$

4. Proteínas virales: se basan en proteínas del propio patógeno, encargadas de la respuesta del sistema inmune de memoria. Son mucho más seguras, pero su capacidad de protección es limitada. ${ }^{3-6}$

5. Vacunas de virus inactivado: estas vacunas se encargan de destruir o inactivar un determinado virus, lo que no permite el desarrollo de la patología. En su tecnología incluyen todo el virión, es decir, una forma infecciosa del virus fuera de la célula huésped, con un núcleo de ácido ribonucleico y una cápside, que se inactiva físicamente por calor o químicamente. Tienen el poder de replicarse in vivo presentando una sintomatología muy leve o nula, estimulando el sistema inmunológico de tal manera que previene la infección. La farmacéutica Sinovac empleó esta metodología para la elaboración de su vacuna. ${ }^{2}$

6. Vectores virales: se modifica el virus, para dar origen a uno del tipo carrier, el mismo que se encarga de transportar la secuencia que recoge la proteína S del COVID-19. En el hospedador se formará una inmunidad de anticuerpos neutralizantes frente al carrier, y por consiguiente la eficacia de la vacuna se ve disminuida. Esta plataforma es usada por las vacunas de AstraZeneca, Sputnik V (Gam-COVID) y la Johnson \& Jonhson de Janssen Pharmaceutical. ${ }^{2-6}$

Las vacunas son una creación del hombre en su intento por frenar el coronavirus, pero, éste en su intento por permanecer ha ido mutando y creando nuevas variantes, las cuales son: ${ }^{3}$

1. B.1.526 y B.1.525: originadas en Nueva York, las mismas que tienen un potencial menor al momento de la neutralización por tratamientos con anticuerpos, suero de convaleciente y de postvacunación. ${ }^{3}$

2. P2: proveniente de Brasil, tiene una disminuida neutralización por tratamientos con anticuerpos, suero de convaleciente y de postvacunación. ${ }^{3}$

3. B.1.1.7: originaria de Reino Unido, es mayormente transmisible (50\%) y la neutralización por tratamientos con anticuerpos, suero de convaleciente y de postvacunación es menor. ${ }^{3}$ 
4. P1: procede de Japón y Brasil, su impacto en la neutralización por tratamiento con anticuerpos es mayor, mientras que por el suero de convaleciente y de postvacunación es menor. ${ }^{3}$

5. B.1.351: encontrada en Sudáfrica, neutralización por tratamiento con anticuerpos es mayor que aquella por el suero de convaleciente y de postvacunación. ${ }^{3}$

6. B.1.427 y B.1.429: originadas en California, su transmisión se ve aumentada en un $20 \%$ y su neutralización por tratamiento con anticuerpos es mayor en comparación al suero de convaleciente y de postvacunación. ${ }^{3}$

Cada una de las vacunas contra el COVID-19 han sido evaluadas por la agencia de Administración de Medicamentos y Alimentos (FDA por sus siglas en inglés) para la autorización y uso de emergencia masiva de las mismas, cada vacuna presenta diferentes tipos de envasado, administración, preparación y almacenamiento, todos estos aspectos son de gran importancia para preparar a los profesionales de atención médica, ya que ellos serán los encargados de recibir y suministrar la vacuna en cada individuo, además el conocimiento de la preservación de cada vacuna es importante para asegurar la eficacia de la misma.?

Las vacunas deben ser no sólo eficaces sino también seguras, y para poder determinarlo se toman en cuenta tres variables: la respuesta inmune, que debe poder enfrentar adecuadamente el agente patógeno; la calidad, es decir cada vacuna debe contener únicamente componentes apropiados, que no pongan en riesgo la vida de las personas; y el seguimiento que se debe dar a las personas a las cuales se les suministró la vacuna para la elaboración de futuros ensayos clínicos. ${ }^{4}$

El desarrollar programas de vacunación es de gran importancia ya que disminuiría significativamente la transmisión del virus y con ello los sistemas de salud no colapsarían. Por lo tanto, se recomienda a la población mundial aplicarse las vacunas, siempre y cuando sean autorizadas por la Organización Mundial de la Salud, haciendo hincapié en la fabricada por Johnson \& Johnson y Pfizer, ya que producen menos reacciones adversas en las personas que las reciben. ${ }^{8}$

\section{VACUNA AD.26.COV2.S}

La vacuna de la multinacional Johnson \& Johnson es del tipo vector adenovirales que basan su creación en la colocación de un gen del DNA propio del virus en el vector viral, el cual se emplea para la transportación del gen a una célula humana, éste se encarga de la codificación de la proteína
S, que es exclusiva de la superficie del SARS-CoV-2. Al llegar a la célula, el vector viral con ayuda de este gen y del mecanismo de la célula para producir la proteína $S$, la ubica en la superficie de la célula, lo cual permitiría la producción de anticuerpos, listos para combatir la infección. ${ }^{2,5}$

Según varios estudios, se ha demostrado que en seis meses del uso de la vacuna en personas mayores a 18 años se podrían prevenir 3,926-9,395 ingresos hospitalarios relacionados con COVID-19, 928-2,236 ingresos en la $\mathrm{UCl}$ y 586-1,435 muertes. Por otra parte, hubo incertidumbre con respecto a la seguridad de la vacuna, puesto que se reportaron seis casos de un efecto secundario poco común como la coagulación sanguínea entre casi siete millones de participantes de la vacuna Janssen. 7,9,10

Embalaje: la vacuna vectorizada por adenovirus de Johnson \& Johnson se envía a una temperatura de -20 ${ }^{\circ} \mathrm{C}$. Se entregará en 10 viales por caja y 48 cajas por caja de envío. Cada embalaje contendrá 2,400 dosis; cada vial contiene cinco dosis de $0.5 \mathrm{~mL}$ ( $2.5 \mathrm{~mL}$ de suspensión en un vial multidosis) en vidrio de tipo I con tapón de goma, precinto de aluminio y cápsula de cierre de plástico azul. La suspensión es incolora, entre transparente y muy opalescente (pH 6-6.4). ${ }^{7}$

Almacenamiento: la vacuna J\&J al cabo de su llegada debe ser transportada inmediatamente al refrigerador y ésta se puede almacenar hasta un periodo de tres meses si se le mantiene en una temperatura de $2-8{ }^{\circ} \mathrm{C} .711$

Preparación: este tipo de vacuna no requiere una preparación especial con respecto a otras. ${ }^{7}$ No contiene adyuvantes, ni antibióticos ni conservantes. ${ }^{3}$ Esta vacuna de Janssen una vez que el vial se perfora en un principio, se puede almacenar en el refrigerador hasta por seis horas, pasado este periodo de tiempo se debe desechar. ${ }^{7}$

Administración: esta vacuna está recomendada para mayores de 18 años y se debe suministrar $0.5 \mathrm{~mL}$ por vía intramuscular a nivel del músculo deltoides. Algunos estudios pueden verificar la necesidad de una inyección de refuerzo después de la primera administrada. $5,7,11,12$

Después de una extensa revisión minuciosa con los datos disponibles, utilizando una escala del 1 al 4, siendo 1 el dato de mayor eficacia y 4 de menor eficacia, los resultados demostraron que los beneficios de la vacuna Janssen fue de tipo 2 (certeza moderada) para la prevención de COVID-19 sintomático, y en cuanto a la prevención de hospitalizaciones y muertes también presentó un valor de 2 (certeza moderada), siendo de gran importancia, ya que se puede administrar en una sola dosis y muy necesaria para la inmunización de personas de difícil acceso, como personas sin hogar y en proceso de rehabilitación, los médicos mencionan que si este 
proceso se pausa sería devastador para los trabajadores de atención de primera línea. ${ }^{9}$

Administración a grupos de población especiales: según un ensayo de fase 3 no es recomendable administrar la vacuna a individuos que presenten hipertensión, cardiopatías graves, obesidad, diabetes o infección por $\mathrm{VIH}$, debido a que el riesgo de presentar COVID-19 grave aumenta notoriamente. A este último grupo de personas se le debe administrar la vacuna sólo si la infección se encuentra controlada por medio de tratamiento antirretrovírico de gran actividad. ${ }^{13}$

Por otro lado, es recomendable suministrar la vacuna en los adultos mayores, ya que el riesgo de presentar COVID-19 y fallecer es alto. En las mujeres que se encuentren en estado de gestación, se aplicará la vacuna únicamente si los beneficios superan los riesgos, ya que no se han encontrado estudios para contraindicar a este grupo de personas. La OMS menciona que no es necesario interrumpir la lactancia materna. ${ }^{13}$

Efectos adversos: los receptores de la vacuna por lo general sintieron síntomas de reactogenicidad, definidas como reacciones adversas sistémicas, por ejemplo cefalea, náuseas, vómito, fatiga, diarreas y un leve dolor en el lugar del pinchazo, no obstante, los síntomas fueron relativamente leves y se resolvieron al cabo de dos días después de la vacunación. ${ }^{7}$

La FDA en abril de 2021 desconfió de Johnson \& Johnson e indicó detener el uso de la vacuna en contra del COVID-19 de Janssen después de que en Estados Unidos se presentaron seis casos de trombosis del seno cavernoso, siendo éste un síndrome tromboembólico inhabitual, por lo que debió ser discutido con respecto a sus beneficios y riesgos por la FDA y concluyeron que se debían considerar las recomendaciones mencionadas en varios estudios actualizados con respecto al uso de la vacuna Janssen en contra del COVID-19 en los Estados Unidos y recomendó el uso provisional en personas mayores de 18 años, advirtiendo que ahora pueden ocurrir episodios de coagulación después de recibir la vacuna, siendo prevalentes las mujeres de 18 a 49 años, también recomendó la advertencia al paciente por partes de los proveedores sobre el riesgo de síndrome de trombosis con trombocitopenia (TTS) con la vacuna Janssen COVID-19, especialmente entre mujeres menores de 50 años. ${ }^{10}$

Uno de los efectos adversos inesperados fue un síndrome de trombocitopenia, específicamente trombosis del seno cavernoso registrándose en un hombre de 25 años con una historia clínica sin antecedentes médicos ni tomaba ninguna medicación, el receptor experimentó síntomas como dolor de cabeza, fatiga, mialgia, fiebre, dolor abdominal, rinorrea y congestión, siendo al día 19 después de recibir la vacuna que el paciente experimenta una convulsión tónica del colon y mediante una tomografía se confirmó una hemorragia cerebral. ${ }^{11}$

Inmunogenicidad: esta vacuna ha logrado neutralizar más de $90 \%$ de anticuerpos en el día 29, mientras que en el día 57 alcanzó $100 \%{ }^{3}$

Eficacia de la vacuna: se refiere a la capacidad que pertenece a la vacuna de prevenir síntomas, gracias a estudios realizados mediante fases de estudios, la vacuna Janssen/Johnson \& Johnson presenta un $66 \%$ de eficacia. ${ }^{13}$

En personas con infección previa de COVID su respuesta inmune no es suficiente ni dura mucho tiempo, por lo que no altera el valor de eficacia de la vacuna, por otro lado, según estudios no existe riesgo o complicaciones para personas que presenten una infección de COVID-19 previo a la vacuna, por lo que se puede decir que no es una contraindicación vacunarse para estas personas. ${ }^{13}$

Estudios previos realizados a primates marcan una notable eficacia del Ad26, donde se evidenció la protección de los pulmones después de seis meses de administrar la primera dosis. ${ }^{14} \mathrm{En}$ el caso de las mujeres lactantes o en etapa de embarazo que reciban la vacuna, es poco probable que transmitan al lactante o feto los efectos adversos de la misma, considerándose segura. ${ }^{15,16} \mathrm{El}$ adenovirus que se libera en el organismo no es capaz de multiplicarse, por lo que no produce una infección. ${ }^{17}$ Varios días después de la inmunización, ya es posible observar los anticuerpos de unión contra la proteína $S$ en la población. ${ }^{18}$

\section{PRECAUCIONES}

A corto plazo, la probabilidad de presentar algún tipo de reacción alérgica por la aplicación de la vacuna es muy baja. Sin embargo, por precaución se pide al paciente que después de la inoculación espere por alrededor de 15 minutos. $3,13,19$

En casos que presenten una temperatura corporal mayor a $38.5^{\circ} \mathrm{C}$ no se debe administrar la vacuna hasta que la fiebre haya desaparecido. No se debe administrar a personas infectadas por COVID-19 hasta que hayan cumplido con los respectivos protocolos de aislamiento. Al contrario, si la persona presenta un resfriado leve esto no debe ser causa para retrasar la vacuna. ${ }^{13}$

Posterior a la inoculación, se debe tomar en cuenta, sobre todo a largo plazo, la posible presencia de trombosis en combinación de trombopenia. Por lo que, a los profesionales de la salud se les recomienda mantener en vigilancia los signos y síntomas de estos pacientes para posterior a ello seguir con un tratamiento adecuado al paciente..$^{20}$ 


\section{REFERENCIAS}

1. Zhao J, Zhao S, Ou J, Zhang J, Lan W, Guan W et al. COVID-19: Coronavirus vaccine development updates. Front Immunol. 2020; 11: 602256. doi: 10.3389/fimmu.2020.602256.

2. Kounis NG, Koniari I, de Gregorio C, Velissaris D, Petalas K, Brinia A et al. Allergic reactions to current available COVID-19 vaccinations: pathophysiology, causality, and therapeutic considerations. Vaccines (Basel). 2021; 9 (3): 221.

3. Picazo J. Vacuna frente al COVID-19. Sociedad Española de Quimioterapia: infección y vacunas. 2019. Disponible en: https:// seq.es/wp-content/uploads/2020/12/vacunas-covid-2.5.pdf

4. Soldevilla PJ, Cardona PJ, Cayla JA, Hernández A, Palma D, Rius C. Revisión sobre las vacunas frente a SARS-CoV-2. Enf Emerg. 2021; 20 (1): 7-1. Disponible en: https://www.uitb.cat/wp-content/ uploads/2021/02/Review-vacunas-COVID_Final_16022021.pdf

5. Gaus D. COVID-19: vacunas. Práctica Familiar Rural. 2021; 6 (1). Disponible en: https://www.practicafamiliarrural.org/index.php/pfr/ article/view/196/226

6. De Francisco ALM. Vacunas SARS-COV2 marzo 2021. Nefrología al día. 2020. Disponible en: https://static.elsevier.es/nefro/ monografias/1/366/366_110320211742.pdf

7. Holm MR, Poland GA. Critical aspects of packaging, storage, preparation, and administration of mRNA and adenovirus-vectored COVID-19 vaccines for optimal efficacy. Vaccine. 2021; 39 (3): 457-459. doi: 10.1016/j.vaccine.2020.12.017.

8. Sadoff J, Gray G, Vandebosch A, Cárdenas V, Shukarev G, Grinsztejn $B$ et al. Safety and efficacy of single-dose Ad26.COV2.s vaccine against Covid-19. N Engl J Med. 2021; 384 (23): 2187-2201. doi: 10.1056/NEJMoa2101544.

9. Ledford $\mathrm{H}$. COVID vaccines and blood clots: five key questions. Nature. 2021; 592 (7855): 495-496. doi: 10.1038/d41586-02100998-w.

10. MacNeil JR, Su JR, Broder KR et al. Recomendaciones actualizadas del Comité Asesor sobre Prácticas de Inmunización para el uso de la vacuna COVID-19 de Janssen (Johnson \& Johnson) después de informes de trombosis con síndrome de trombocitopenia entre receptores de la vacuna - Estados Unidos, abril de 2021. MMWR Morb Mortal Wkly Rep. 2021; 70: 651-656. Disponible en: http:// dx.doi.org/10.15585/mmwr.mm7017e4

11. Janssen Biotech, Inc. (2021, 26 febrero). FDA Briefing Document Janssen Ad26.COV2.S Vaccine for the Prevention of COVID-19. Vaccines and Related Biological Products Advisory Committee Meeting February 26, 2021. Available in: https://www.fda.gov/ media/146217/download
12. Oliver SE, Gargano JW, Scobie H, Wallace M, Hadler SC, Leung J et al. The advisory committee on immunization practices' interim recommendation for use of Janssen COVID-19 vaccine - United States, February 2021. MMWR Morb Mortal Wkly Rep. 2021; 70 (9): 329-332. doi: 10.15585/mmwr.mm7009e4.

13. Organización Mundial de la Salud. Vacuna de Janssen contra la COVID-19. Ginebra, Suiza: OMS; 2021.

14. Roozendaal R, Solforosi L, Stieh D, Serroyen J, Straetemans R, Wegmann $\mathrm{F}$ et al. SARS-CoV-2 binding and neutralizing antibody levels after vaccination with Ad26.COV2.S predict durable protection in rhesus macaques. bioRxiv J. 2021. Avaialbe in: https:// doi.org/10.1101/2021.01.30.428921

15. Drugs and Lactation Database (LactMed). Bethesda (MD): National Library of Medicine (US); 2021. COVID-19 vaccines. Available in: https://www.ncbi.nlm.nih.gov/books/NBK565969/

16. D'Amico F, Rabaud C, Peyrin-Biroulet L, Danese S. SARS-CoV-2 vaccination in IBD: more pros than cons. Nat Rev Gastroenterol Hepatol. 2021; 18 (4): 211-213. doi: 10.1038/s41575-02100420-w.

17. Livingston EH, Malani PN, Creech CB. The Johnson \& Johnson vaccine for COVID-19. JAMA. 2021; 325 (15): 1575. doi: 10.1001/ jama.2021.2927.

18. Stephenson KE, Le Gars M, Sadoff J, de Groot AM, Heerwegh D, Truyers $C$ et al. Immunogenicity of the Ad26.COV2.S vaccine for COVID-19. JAMA. 2021; 325 (15): 1535-1544.

19. Jhaveri $R$. The next set of COVID-19 vaccines: leveraging new development platforms to increase access for more people around the world. Clin Ther. 2021; 43 (4): 702-710.

20. MUH (FV). Vacuna frente a la COVID-19 de Janssen: conclusiones de la evaluación del riesgo de trombosis junto con trombocitopenia: Conclusiones del comité para la evaluación de riesgos en farmacovigilancia Europeo. Agencia española de medicamentos y productos sanitarios. 07/2021. Disponible en: https://www.aemps. gob.es/informa/notasInformativas/medicamentosUsoHumano/ seguridad/2021/docs/NI_MUH_FV-07-2021-Janssen.pdf?x58195

Conflicto de intereses: Los autores declaran no tener conflicto de intereses.

Financiamiento: Autofinanciado.

Correspondencia:

Diana Patricia Álvarez-Álvarez

E-mail: dalvareza@ucacue.edu.ec 